\title{
Synergistic effect of regenerating agent plus cord blood serum eye drops for the treatment of resistant neurotrophic keratitis: a case report and a hypothesis for pathophysiologic mechanism
}

\author{
This article was published in the following Dove Press journal: \\ International Medical Case Reports Journal \\ 2 November 2015 \\ Number of times this article has been viewed
}

\author{
Giuseppe Giannaccare \\ Michela Fresina' \\ Aldo Vagge ${ }^{2}$ \\ Piera Versura' \\ 'Ophthalmology Unit, DIMES, \\ S Orsola-Malpighi Teaching Hospital, \\ University of Bologna, Bologna, Italy; \\ ${ }^{2}$ Eye Clinic, DiNOGMI, University of \\ Genoa, Genoa, Italy
}

\begin{abstract}
This report describes a case of a 72-year-old Caucasian woman presenting with a large neurotrophic keratitis with a large persistent epithelial defect, with a longest linear diameter of $7 \mathrm{~mm}$ and greatest perpendicular width of $5 \mathrm{~mm}$, affecting epithelium, Bowman membrane, and anterior stroma. Corneal disease was resistant to conventional treatment and classified as stage 2 according to Mackie classification. Patient was instructed to instill regenerating agent (RGTA) eye drops in the morning, as the first eye drop, once every 5 days in combination with daily cord blood serum (CBS) eye drops 6 times/day. The patient was asked to visit after 1 week (V1), 2 weeks (V2), 3 weeks (V3), and 4 weeks (V4) of study treatment. In V4 treatment, corneal sensitivity improved, and keratitis healed with resolution of stromal inflammation. Global treatment tolerance was very satisfactory. Patient continued the therapy for a further month after complete healing. Currently, the patient has been followed up for 3 months without any sign of keratitis recurrence. To the best of our knowledge, this case report describes for the first time the successful combined use of RGTA and CBS eye drops for the treatment of neurotrophic keratitis resistant to conventional treatment. We hypothesize that RGTA eye drops could provide an optimal migration substrate and that growth factors supplied by CBS eye drops could strengthen the repair process by promoting cell growth over the matrix. Combined RGTA and CBS eye drop therapy could be a new potential option for the successful treatment of resistant neurotrophic keratitis, particularly when each drug alone is not effective.
\end{abstract}

Keywords: neurotrophic keratitis, regenerating agent, cord blood serum eye drops, penetrating keratoplasty, in vivo confocal microscopy

\section{Background}

Neurotrophic keratitis is a degenerative disease caused by damage of trigeminal innervation leading to corneal epithelial breakdown, impairment of healing and, in severe cases, corneal ulceration, melting, and perforation. The hallmark of this disease is a decrease or absence of corneal sensation. ${ }^{1}$ The trigeminal nerve provides not only corneal sensation but also trophic factors to the cornea, playing a key role in maintaining the anatomical integrity and functioning of the entire ocular surface. ${ }^{2}$ Several ocular and systemic diseases can affect the trigeminal nerve at different levels. The most common causes include herpetic keratitis, chemical burns, physical injuries, and corneal surgery. Intracranial space-occupying lesions such as neuroma, meningioma, and aneurysms may also lead to a compression of the trigeminal nerve or ganglion and produce an impairment of corneal sensitivity. ${ }^{3}$ Systemic diseases such as diabetes and multiple sclerosis may decrease sensory nerve function or damage sensory fibers leading to corneal hypoesthesia. ${ }^{4}$ The
Correspondence: Giuseppe Giannaccare Ophthalmology Unit, DIMES, S Orsola-Malpighi Teaching Hospital, University of Bologna, Via Pelagio Palagi n 9, 40138 Bologna, Italy Email giuseppe.giannaccare@gmail.com 
diagnosis is mainly based on the clinical history, presence of persistent epithelial defects or ulcers, and decreased corneal sensitivity. Patients rarely complain of ocular symptoms, and there is a significant discrepancy between clinical findings and subjective symptoms. A neurotrophic keratitis classification based on severity was proposed by Mackie, who distinguished three stages: epithelial alterations (stage 1), persistent epithelial defect (stage 2), and corneal ulcer (stage 3). ${ }^{5}$

Although the clinical diagnosis is easily oriented from clinical history and findings, the pharmacological management for this disease is one of the most challenging among all other ophthalmological diseases. Withdrawal of all topical drugs is always required because they have detrimental effects on the ocular surface epithelium. Preservative-free artificial tears may improve ocular surface health at all stages of disease severity. In severe cases, the use of topical antibiotic eye drops is recommended to prevent infections. Steroids may increase the risk of corneal melting and perforation by inhibiting stromal healing, and their use should be considered with great caution. Nonsteroidal anti-inflammatory eye drops may inhibit the healing process and should be avoided. Several studies, including case reports, case series, and clinical trials, have recently reported the efficacy of different bloodderived eye drops in promoting neurotrophic keratitis healing because of their high concentrations of growth factors that are essential in wound healing. These products include eye drops obtained from autologous serum, cord blood serum (CBS), and platelet rich plasma. ${ }^{6-8}$ Furthermore, three Phase II clinical trials are currently ongoing to evaluate the safety and efficacy of novel therapies for patients suffering from neurotrophic keratitis, including recombinant human nerve growth factor and regenerating agent (RGTA; Cacicol20, OTR3, Paris, France) (ClinicalTrials.gov identifiers NCT02227147, NCT01756456, and NCT01794312). ${ }^{9-11}$

CBS eye drops contain biologically active components and, in particular, growth factors essential in corneal homeostasis and wound healing, and work by regulating cellular proliferation, differentiation, and migration. ${ }^{12} \mathrm{CBS}$ eye drops were shown to be more effective in treating severe dry eye syndrome compared with autologous serum eye drops. ${ }^{13}$ Furthermore, in vitro studies showed that RGTAs may act as survival and protective agents in several pathological tissue injuries, mimicking the action of destroyed heparan sulfate (HS) molecules, protecting the bioavailability of preexisting and newly synthesized growth factors, and recreating a matrix microenvironment in which cells can migrate and multiply. ${ }^{12,13}$

Taking into account these laboratory results, we hypothesized a theoretical synergistic effect of RGTA and CBS eye drops at the site of injury and tried to translate this theory to clinical practice by treating, for the first time, a case of neurotrophic keratitis resistant to conventional therapy with a combination of RGTA and CBS eye drops rich in growth factors.

\section{Case presentation}

A 72-year-old Caucasian woman was referred to our emergency ward with progressive loss of vision in her left eye. The patient also observed the appearance of a wide white spot in the center of the cornea from a few days (Figure 1). About her clinical history, 6 months before she underwent tectonic penetrating keratoplasty in the same eye for the treatment of severe neurotrophic keratitis, which was the first clinical sign of a Gasser ganglion neuroma. The corneal disease worsened after endoscopic surgical removal of intracranial lesion and required a first corneal transplant.

In the emergency ward, we performed complete ophthalmologic examination and admitted the patient to the hospital, prescribing systemic (meropenem intravenous) and topical broad-spectrum antibiotic therapy (fortified vancomycin and tobramycin eye drops every hour) and scheduling a second corneal transplant. Corneal graft melting progressed to perforation with iris prolapse in 2 days (Figure 2).

We performed an emergency keratoplasty (tectonic large diameter $11 \mathrm{~mm}$ penetrating) because only the extreme perilimbal periphery of recipient cornea was spared by the melting. We used interrupted 10/0 monofilament nylon sutures, and the procedure was associated with iris reconstruction, extracapsular extraction of intumescent lens, and chemodenervation of levator palpebrae superioris with $5 \mathrm{U}$ of botulinum toxin type-A to induce temporary ptosis for corneal graft protection. Two days after surgery, the patient

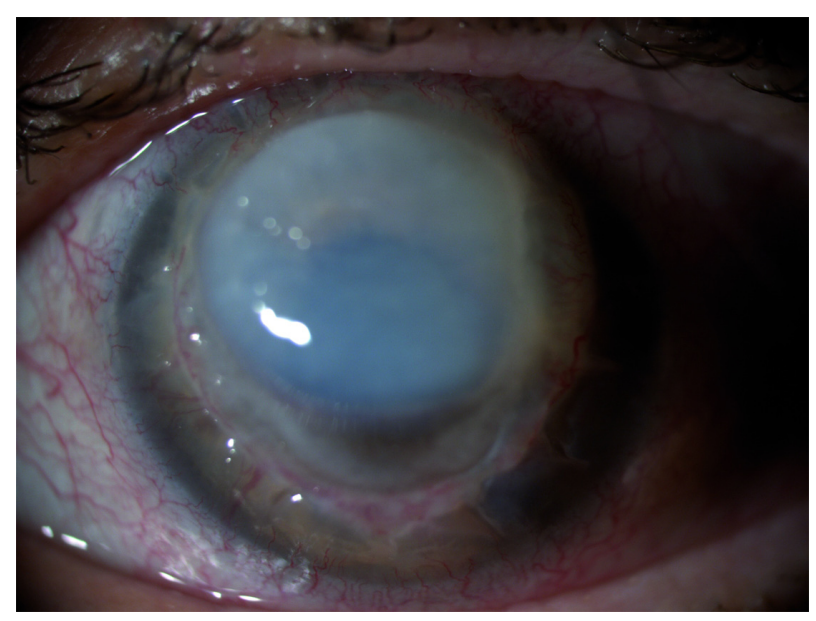

Figure I Corneal graft melting.

Note: Corneal graft melting observed when patient came to the emergency ward. 


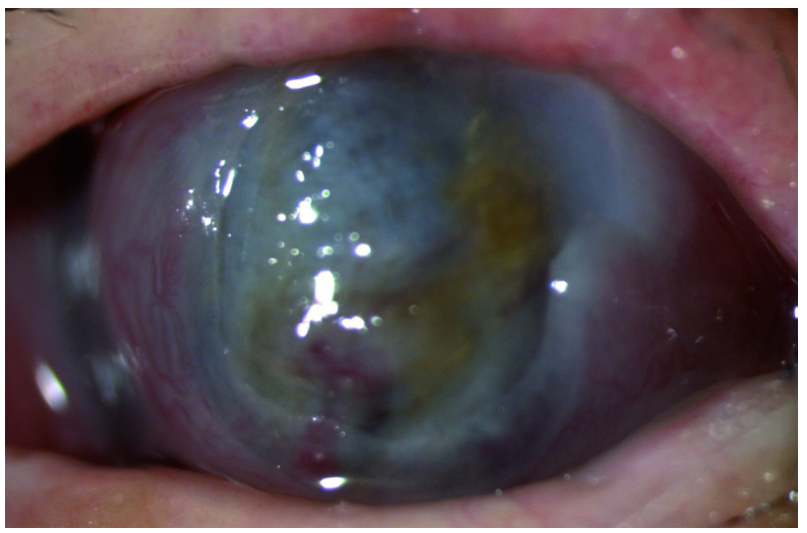

Figure 2 Corneal graft perforation with iris prolapse.

Note: Corneal graft melting rapidly progressed to corneal perforation with iris prolapse although given the systemic and topic broad-spectrum antibiotic therapy.

was discharged from the hospital with systemic and topical steroids and preservative-free tear substitutes. The patient was visited once a week for regular checks during the postoperative course. At the third postoperative month a neurotrophic keratitis appeared. Corneal photography at a magnification of $\times 16$ showed that a large persistent epithelial defect with longest linear diameter equal to $7 \mathrm{~mm}$, greatest perpendicular width equal to $5 \mathrm{~mm}$, and smooth, rolled edges (Figure 3 ). No signs of ocular inflammation were present. Neurotrophic keratitis was classified as stage 2 according to the Mackie classification. Confocal microscopy analysis showed no nerve regeneration and stromal inflammation in the corneal graft (Figure 4). Corneal sensitivity measured by Cochet-Bonnet aesthesiometer in the standardized five regions of corneal graft was equal to $0 \mathrm{~mm}$ (length filament extrusion). The keratitis was sterile on microbiologic examination and did

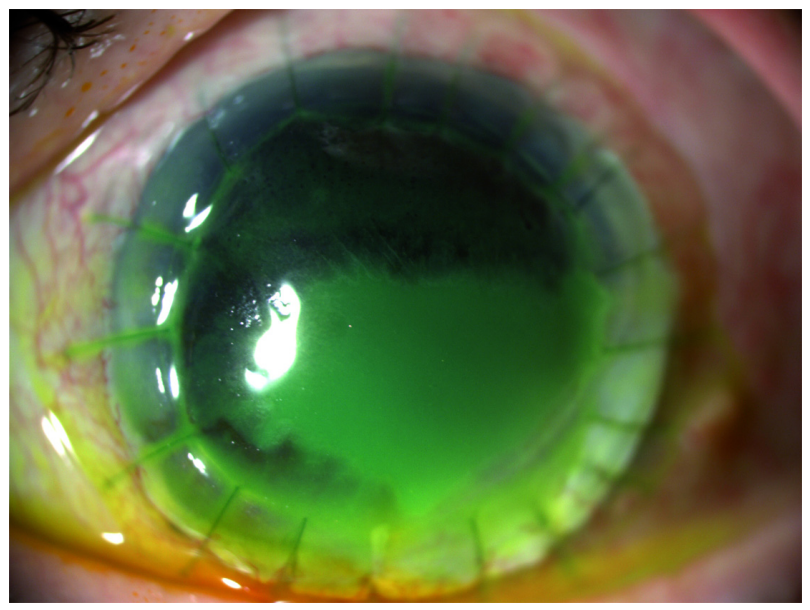

Figure 3 Corneal ulcer before study treatment.

Note: Corneal ulcer affected epithelium, Bowman membrane, and anterior stroma with a longest linear diameter equal to $7 \mathrm{~mm}$ and a greatest perpendicular width equal to $5 \mathrm{~mm}$.

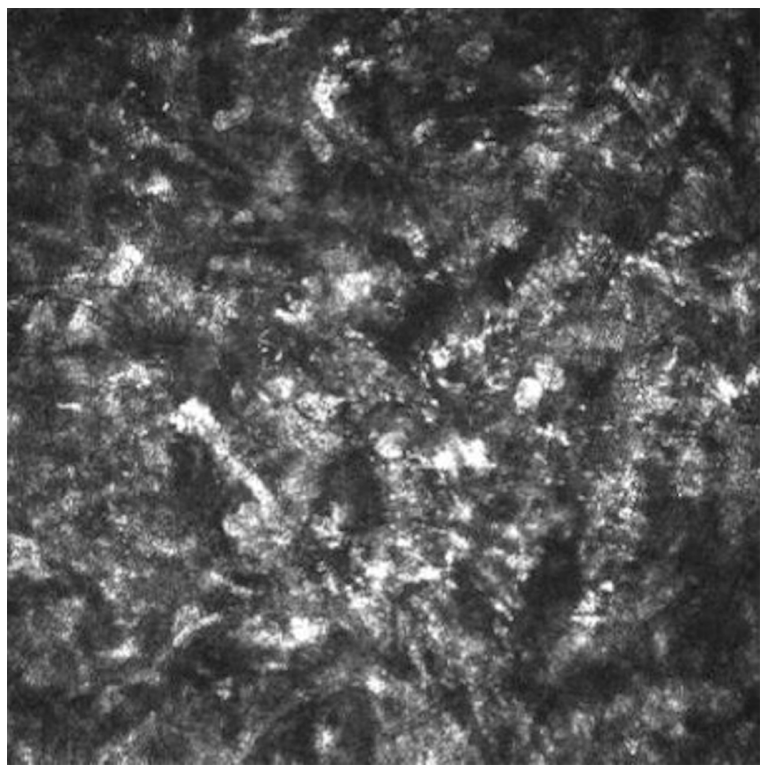

Figure 4 Confocal microscopy analysis of corneal ulcer before study treatment. Note: Confocal microscopy analysis showed no nerve regeneration and stromal inflammation in corneal graft.

not improve after 4 weeks of conventional treatment, including discontinuation of all epitheliotoxic topical medication and frequent (every 2 hours) instillation of preservative-free tear substitutes.

Taking into account previous keratoplasty failure, total corneal anesthesia, keratitis large size, and resistance to conventional treatment, we decided to prescribe RGTA in association with CBS eye drops. Institutional Review Board approval was not deemed necessary for this off label treatment. However all principles outlined in the Declaration of Helsinki were followed. The patient was instructed to instill RGTA eye drops in the morning, as the first eye drop, once every 5 days in combination with daily CBS eye drops six times/day. The patient was visited after 1 week (V1), 2 weeks (V2), 3 weeks (V3), and 4 weeks (V4) of study treatment. In each follow-up visit, all the aforementioned procedures were repeated. In detail, corneal photographies showed partial healing $>50 \%$ already in V2 (Figure 5) and a complete healing in V4 (Figure 6). Confocal microscopy showed complete resolution of stromal inflammation in V4 (Figure 7). Cochet-Bonnet aesthesiometry showed a mild improvement in V4 compared with baseline ( $10 \mathrm{~mm}$ vs $0 \mathrm{~mm}$ length filament extrusion). The mean palpebral fissure height of approximately $3.5 \mathrm{~mm}$ persisted over time during treatment period allowing both corneal protection and a better ocular surface examination compared to tarsorrhaphy. Treatment global tolerance assessed by both examiners and patient was very satisfactory. No adverse events occurred. 


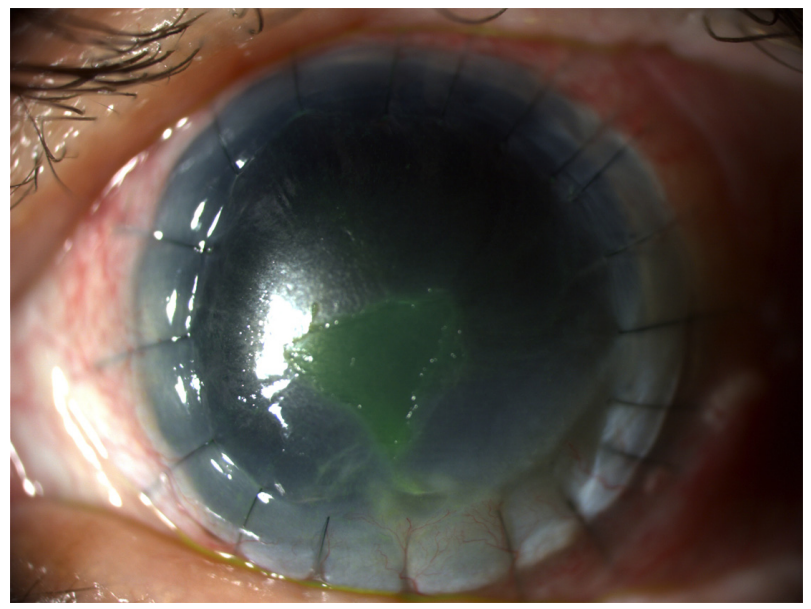

Figure 5 Corneal ulcer after 2 weeks of study treatment (V2).

Note: Corneal ulcer improved after therapy with a partial healing $>50 \%$ already in V2.

Patient continued the therapy for a further month after complete healing (total treatment duration 8 weeks), after which she stopped and used only preservative-free tear substitutes.

Currently, the patient has been followed up for 3 months without any sign of keratitis recurrence. Written informed consent was obtained from the patient for publication of this case report and any accompanying images.

\section{Discussion}

Neurotrophic keratitis is a degenerative corneal disease caused by impairment of trigeminal innervation. Although the clinical diagnosis is easily oriented from the clinical history and findings, the pharmacological management represents a real therapeutic challenge for ophthalmologists, particularly in patients with total corneal anesthesia as in the case presented earlier. Several therapeutic approaches have been

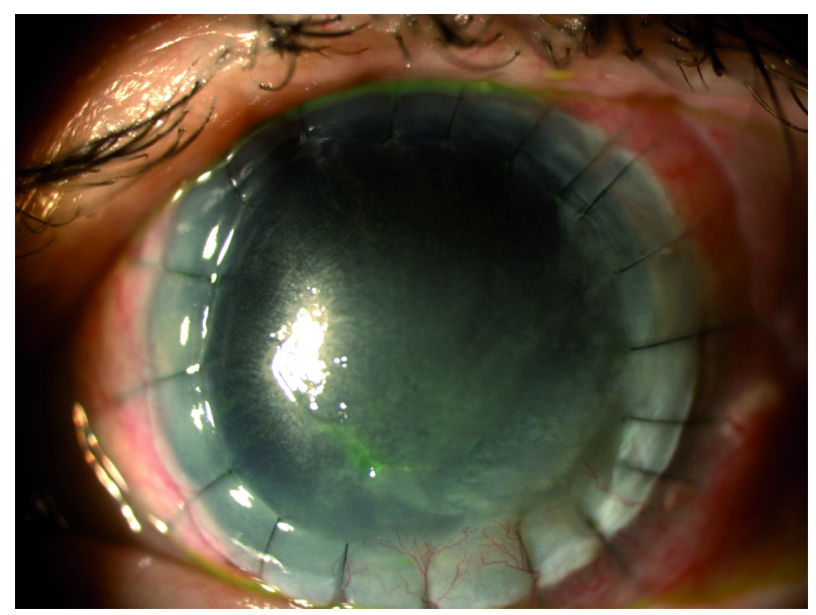

Figure 6 Corneal ulcer healing after 4 weeks of study treatment (V4). Note: Corneal ulcer completely healed in V4.

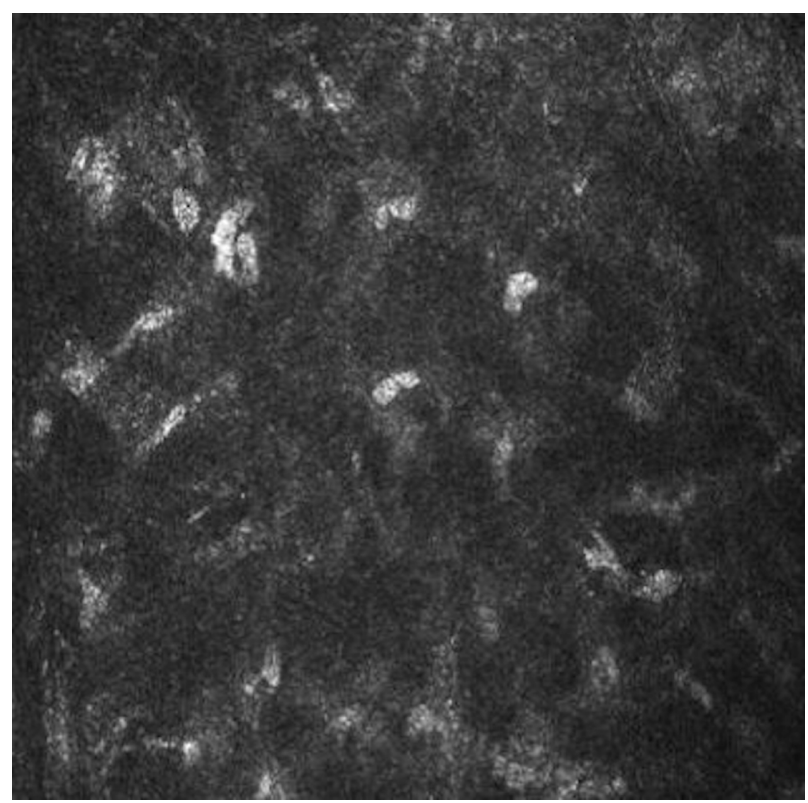

Figure 7 Confocal microscopy analysis of the cornea after 4 weeks of study treatment (V4).

Note: Confocal miscroscopy showed complete resolution of stromal inflammation in $\mathrm{V} 4$.

proposed to halt the progression of the disease and to avoid corneal perforation. Corneal healing is a complex process involving cellular interaction and various molecules such as proteases, growth factors, and epithelial and stromal cytokines. To the best of our knowledge, this case report describes for the first time the successful combined use of RGTA and CBS eye drops for the treatment of neurotrophic keratitis resistant to conventional treatment.

RGTAs constitute a new class of medicinal substances that enhance both speed and quality of tissue healing and may lead to a real tissue regenerating process. RGTAs consist of chemically engineered polymers adapted to interact with and protect against proteolytic degradation of cellular signaling proteins such as growth factors, cytokines, interleukins, colony-stimulating factors, chemokines, and neurotrophic factors. Indeed, almost all these proteins of cellular communication are naturally stored in the extracellular matrix, interacting specifically with the HSs. After any tissue injury, dead cells release glycanases and proteases inducing HS degradation first, and subsequently, that of the cytokines, which are no longer protected. ${ }^{14,15}$

CBS is obtained from umbilical vein at delivery and contains many growth factors (epidermal growth factor, transforming growth factor- $\beta$, insulin-like growth factor 1 , nerve growth factor) and essential tear components such as vitamin A and substance P. CBS can be used effectively in many ocular surface diseases, including severe dry eye 
syndrome, persistent epithelial defects, and neurotrophic keratitis. $^{12,13}$

The explanation for the synergistic effect of combined RGTA and CBS eye drops therapy originates from the hypothesis that in injured tissues RGTA eye drops could replace the natural HS and provide an optimal migration substrate, whereas growth factors supplied by CBS eye drops could strengthen the repair process by promoting cell growth over the regenerated matrix.

\section{Conclusion}

Neurotrophic keratitis can cause severe visual impairment and its treatment is very challenging for ophthalmologists. No therapies are currently available to restore corneal innervation and visual acuity. This case report describes for the first time the successful combined use of RGTA and CBS eye drops for the treatment of neurotrophic keratitis resistant to conventional treatment. Combined therapy with RGTA and CBS eye drops could be a new potential option for the treatment of neurotrophic keratitis, particularly when each drug alone is not effective. However, the findings of ongoing clinical trials and other translational researches from experimental studies are necessary to enable new potential therapies to treat this orphan disease.

\section{Author contributions}

All authors made substantial contributions to conception and design, acquisition of data, or analysis and interpretation of data; took part in either drafting the article or revising it critically for important intellectual content; gave final approval of the version to be published; and agree to be accountable for all aspects of the work in ensuring that questions related to the accuracy or integrity of any part of the work are appropriately investigated and resolved.

\section{Disclosure}

The authors report no conflicts of interest in this work.

\section{References}

1. Sacchetti M, Lambiase A. Diagnosis and management of neurotrophic keratitis. Clin Ophthalmol. 2014;8:571-579.

2. Müller LJ, Marfurt CF, Kruse F, Tervo TM. Corneal nerves: structure, contents and function. Exp Eye Res. 2003;76:521-542.

3. Puca A, Meglio M, Vari R, Tamburrini G, Tancredi A. Evaluation of fifth nerve dysfunction in 136 patients with middle and posterior cranial fossae. Eur Neurol. 1995;35:33-37.

4. Hyndiuk RA, Kazarian EL, Schultz RO, Seideman S. Neurotrophic corneal ulcers in diabetes mellitus. Arch Ophthalmol. 1977;95: 2193-2196.

5. Mackie IA. Neuroparalytic keratitis. In: Frauenfelder F, Roy FH, Meyer SM, editors. Current Ocular Therapy. Philadelphia, PA: WB Saunders; 1995.

6. Jeng BH, Dupps WJ Jr. Autologous serum $50 \%$ eye drops in the treatment of persistent corneal epithelial defects. Cornea. 2009;28:1104-1108.

7. Geremicca W, Fonte C, Vecchio S. Blood components for topical use in tissue regeneration: evaluation of corneal lesions treated with platelet lysate and considerations on repair mechanisms. Blood Transfus. 2010;8:107-112.

8. Yoon KC, You IC, Im SK, Jeong TS, Park YG, Choi J. Application of umbilical cord serum eyedrops for the treatment of neurotrophic keratitis. Ophthalmology. 2007;114:1637-1642.

9. Dompé SpA. Evaluation of efficacy of $20 \mu \mathrm{g} / \mathrm{ml} \mathrm{rhNGF}$ new formulation (with anti-oxidant) in patients with stage 2 and 3 neurotrophic keratitis. Available from: http://clinicaltrials.gov/show/NCT02227147. NLM identifier: NCT02227147. Accessed September 28, 2014.

10. Dompé S.p.A. Evaluation of safety and efficacy of rhNGF in patients with stage 2 and 3 neurotrophic keratitis. (REPARO). Available from: http://clinicaltrials.gov/show/NCT01756456. NLM identifier: NCT01756456. Accessed September 28, 2014.

11. Laboratoires Thea. Efficacy and safety assessment of T4020 versus vehicule in patients with chronic neurotrophic keratitis or corneal ulcer. Available from: http://clinicaltrials.gov/ct2/show/NCT01794312. NLM identifier: NCT01794312. Accessed September 28, 2014.

12. Versura P, Profazio V, Buzzi M, et al. Efficacy of standardized and quality-controlled cord blood serum eye drop therapy in the healing of severe corneal epithelial damage in dry eye. Cornea. 2013;32: 412-418.

13. Yoon KC, Heo H, Im SK, You IC, Kim YH, Park YG. Comparison of autologous serum and umbilical cord serum eye drops for dry eye syndrome. Am J Ophthalmol. 2007;144:86-92.

14. Rouet V, Hamma-Kourbali Y, Petit E, et al. A synthetic glycosaminoglycan mimetic bind vascular endothelial growth factor and modulates angiogenesis. J Biol Chem. 2005;280:32792-32800.

15. Alexakis C, Mestries P, Garcia S, et al. Structurally different RGTAs modulate collagen-type expression by cultured aortic smooth muscle cells via different pathways involving fibroblast growth factor-2 or transforming growth factor- $\beta 1$. FASEB J. 2004;18:1147-1149.
International Medical Case Reports Journal

\section{Publish your work in this journal}

The International Medical Case Reports Journal is an international, peer-reviewed open-access journal publishing original case reports from all medical specialties. Previously unpublished medical posters are also accepted relating to any area of clinical or preclinical science. Submissions should not normally exceed 2,000 words or

\section{Dovepress}

4 published pages including figures, diagrams and references. The manuscript management system is completely online and includes a very quick and fair peer-review system, which is all easy to use. Visit http://www.dovepress.com/testimonials.php to read real quotes from published authors. 